\title{
REVISIONES
}

\section{Políticas de conservación en Brasil y la Unión Europea: mismos objetivos, diferentes problemas}

\author{
Conservation policies in Brazil and the European Union: \\ same objectives, different problems
}

\begin{abstract}
Manuel Carballal ${ }^{\text {a*, }}$ José Ambrosio Ferreira $^{\text {a }}$, Marcelo Leles Romarco a
*Autor de correspondencia: ${ }^{a}$ Universidade Federal de Viçosa, Departamento de Economía Rural, Avenida Pudue, s/nº, Campus Universitario, edificio Edson Potsch Magalhães, Viçosa, Brasil, tel.: +553138992215, wmanumanu@gmail.com
\end{abstract}

\author{
SUMMARY
}

Nature's conservation has been associated with the dispute of territory from its origins till today. Reducing the limits of property rights and dynamics of use and occupation have been the most widely used techniques to achieve a clear objective as is the preservation of areas with high environmental value. The concept of ecology and conservation has evolved along the last years, starting from the elimination of the human presence in delimited areas. Therefore, it has passed from limiting human presence in delimited areas to coexistence between humans and environment. The most used tool for nature's preservation is the creation of conservation areas, though these have always been associated with the generation of conflicts and problems. In this paper, two conservation models possessing the same objective although using different methods, such as conservation areas of sustainable use in Brazil and the European Union, were compared. Consequently, it explains what they are and describes the problems and conflicts they generate or may generate. Subsequently, a comparison of the two models was made, where the advantages and disadvantages of each one were obtained. Finally, it is concluded that environmental policies should be based on the participation of key players and the existence of lack of information may lead to a rejection of environmental policies.

Key words: conflict, conservation units, ecology, Natura 2000.

\section{RESUMEN}

Desde sus orígenes hasta la actualidad la conservación de la naturaleza está asociada a la disputa del territorio. La acotación de los límites de los derechos de propiedad, de las dinámicas de uso y la ocupación del suelo son las técnicas más empleadas para conseguir la conservación en zonas con alto valor ambiental. El concepto de conservación ha evolucionado a lo largo de los últimos años. Así, se ha pasado de la limitación de la presencia humana en áreas delimitadas a una convivencia respetuosa entre el ser humano y el medio ambiente. La herramienta más empleada para la preservación de la naturaleza es la creación de áreas de conservación, pero estas han estado asociadas habitualmente a la generación de conflictos. En el presente trabajo se comparan dos modelos de conservación que comparten el mismo objetivo pero que emplean aproximaciones diferentes: las áreas de conservación de uso sostenible en Brasil y la Red Natura 2000 de la Unión Europea. Así, se explica en qué consisten y además se describen los problemas y conflictos que generaron o pueden generar. Posteriormente, se realiza una comparación de ambos modelos y se detallan las ventajas e inconvenientes de cada uno. Finalmente se concluye que las políticas ambientales deben estar basadas en la participación de los actores principales y que la falta de información puede llevar un rechazo de las políticas ambientales.

Palabras clave: conflicto, unidades de conservación, ecología, Red Natura 2000.

\section{INTRODUCCIÓN}

Históricamente la ocupación del territorio está asociada a conflictos motivados por la existencia de diferentes intereses: el crecimiento urbanístico, la creación de infraestructuras, la intensificación agraria, entre otros, y destacando entre todos ellos la conservación. Por ello, una de las herramientas más empleada por los países, con el objetivo de la preservación de la naturaleza, son las áreas de conservación. Estas están relacionadas con la acotación de los límites de la propiedad y el cambio en las dinámicas de uso y la ocupación del territorio, con el fin de preservar zonas con alto valor ambiental.

La primera corriente ecologista predominante relacionada con la preservación a nivel mundial fue el naturalismo. Esta corriente disponía de un único método simple 
para proteger el medio ambiente, que consistía en la limitación de la presencia del ser humano en áreas de interés ecológico. El naturalismo fomentaba la creación de espacios naturales protegidos con una ausencia total de actividad humana sobre los espacios a conservar, reservando estas áreas para la investigación científica, el ocio y el turismo, donde se puede observar y sentir lo que es la naturaleza (Diegues 2000). Esta cultura de proteccionismo nació en Estados Unidos y rápidamente traspasó fronteras. En Europa se implantó y se aceptó como algo necesario. En Brasil, al disponer de una gran superficie forestal de alto interés y al estar estas incluidas en acuerdos tanto económicos como políticos, los dirigentes se vieron obligados a crear medidas ambientales (Corrêa 2006).

En todo caso, en los países de América del Sur, la situación ecológica, social y cultural es diferente de la de Estados Unidos. Las zonas a preservar están ocupadas por indígenas, extrativistas ${ }^{1}$ u otras poblaciones tradicionales. Esta realidad genera grandes conflictos por el uso de la tierra, ya que en muchas ocasiones obliga a estas poblaciones a emigrar, algo que no sucede tanto en los Estados Unidos como en algunas zonas de Europa.

Las primeras áreas de conservación creadas en Brasil, generaron numerosos problemas de carácter político, social y económico. Se caracterizan por imponer una nula presencia de poblaciones humanas, dando lugar a la emigración forzosa de las poblaciones y a la exclusión del acceso a los bienes y servicios existentes (Vivacqua y Freire 2007). A mayores hay que destacar la falta de control por parte del gobierno brasileño y el aprovechamiento tanto de las empresas madereras como de explotación minera, que de forma ilegal se apropian de los recursos naturales de estas áreas, dando como resultado un agravamiento de los conflictos (Diegues 2000).

A partir de los años 1960 y 1970, se produce un cambio de percepción y aptitud en la forma de entender la conservación del medio ambiente, a raíz de la cumbre de Estocolmo de 1972, donde los países de la Comunidad Económica Europea (futura Unión Europea) deciden mejorar las políticas ambientales. Comprueban que la técnica de conservación empleada hasta este momento es altamente cuestionada por la sociedad, y deducen que la expulsión de la población de sus áreas de residencia y de fuente de recursos no es la solución. Aislar las áreas a proteger de la población no da como resultado un mundo mejor. Un uso sostenible de los recursos junto a un sistema tradicional de vida es, a veces, mejor que una protección pura, visto desde el punto de vista social y económico.

Tras la publicación de MacArthur y Wilson (1967), sobre la biogeografía, se demuestra que cuanto menor es la superficie de las áreas de conservación mayor es la probabilidad de extinción de los seres vivos a proteger. Pero el

El nombre correcto en español sería recolector, pero se decidió mantener el nombre en portugués al ser un grupo social de alta importancia en las políticas de conservación en Brasil. tamaño no es el único problema, al estar estas islas o áreas de conservación en medio de paisajes modificados por los seres humanos, provocando una fragmentación del hábitat. Ante el aislamiento que sufren estas islas artificiales y tras el estudio del medio, los biólogos recomiendan la creación de corredores ecológicos para facilitar el desplazamiento de los animales y los seres vivos (Bennet 2004). Esto mejora el intercambio y el desplazamiento de las especies sin la necesidad de aumentar la superficie a conservar. El aumento de la movilidad de las especies entre las diferentes reservas y parques (Brunelle y Goodwin 2013), ayuda a eliminar la idea de crear grandes áreas de protección.

El presente trabajo tiene como objetivo demostrar que el empleo de políticas de conservación sostenible puede estar basado en diferentes corrientes ambientales y tener un mismo objetivo.

Las políticas de conservación que se contemplan en este estudio son aquellas utilizadas en Brasil y en la Unión Europea. Más concretamente, la Red Natura 2000 (Red Natura), para el caso de la Unión Europea, y las Unidades de Conservación de Uso Sostenible, destacando las Reservas Extrativistas, para el caso de Brasil. Para obtener el objetivo marcado, primeramente, se realiza un resumen de la evolución de las medidas ambientales a escala mundial, y se muestra cómo para el caso brasileño es, en un primer momento, una política creada ante las presiones internacionales que deriva en un proceso socio-ambiental; mientras que en el caso europeo es desde el principio un proceso ambiental. Acto seguido, se discuten las diferentes políticas empleadas, tanto en Brasil como en la Unión Europea, y los problemas y conflictos que generan. Finalmente, se exponen las ventajas e inconvenientes de ambos modelos y se obtienen unas conclusiones.

\section{CONCEPTO DE CONSERVACIÓN, MÉTODOS EMPLEADOS}

Los métodos empleados que tienen como objetivo la conservación de la naturaleza han variado con el paso de los años. Antes de comenzar a describir su evolución, es importante definir lo que se entiende por conservación tanto en Brasil como en la Unión Europea. La ley que define el concepto de conservación en Brasil es la "Lei $N^{o} 9.985$ de Junho de 2000". Define a la conservación de la naturaleza como el uso realizado por el ser humano, teniendo en cuenta la preservación, la manutención, el empleo sostenible, la restauración y la recuperación del medio ambiente, para poder producir un mayor beneficio, en base sostenible, para las actuales generaciones, preservando y garantizando su potencial para satisfacer las necesidades y aspiraciones de las generaciones futuras, así como la supervivencia de los seres vivos en general (Brasil 2000). Por otro lado la "Directiva 92/43 CEE", que regula la Red Natura, define la conservación como el conjunto de medidas necesarias para mantener o restablecer los hábitats naturales y las poblaciones de especies de fauna y de flo- 
ra silvestre. A mayores define el estado de conservación de un hábitat como el conjunto de influencias que actúan sobre el hábitat natural y sobre las especies típicas asentadas en el mismo y que pueden afectar a largo plazo a su distribución natural, su estructura y funciones así como la supervivencia de la especie en un área geográfica definida y claramente delimitada (CCE 1992).

El primer parque natural creado en todo el mundo, fue el de Yellowstone en los Estados Unidos a mediados del siglo XIX, más concretamente el 1 de marzo de 1872 . Yellowstone fue el resultado de las presiones ejercidas por los movimientos ambientales sobre el gobierno en los Estados Unidos. La perspectiva ambiental dominante en esa época era conocida como preservacionista, es decir, una conservación que limitaba la presencia del ser humano, por no decir una conservación basada en la creación de islas naturales de alto valor estético, que servían para el deleite de la población en sus momentos de ocio. Es importante destacar que los primeros parques naturales creados en el mundo eran áreas con una nula presencia de población. Este dato es fundamental para entender el motivo de rechazo de este modelo de conservación en países en desarrollo, donde las áreas a conservar eran creadas en zonas donde habitaban poblaciones, ya sean indígenas, poblaciones tradicionales o recientemente asentadas (Extrativistas). La creación de diferentes áreas de conservación en países como Brasil, trajo consigo efectos devastadores sobre las poblaciones existentes, al verse obligadas a emigrar a núcleos urbanos y abandonar los territorios donde históricamente vivían (Diegues 2000).

La primera cumbre sobre el medio ambiente se celebra en Estocolmo en 1972. Entre los mayores logros conseguidos en la misma destaca la mayor implicación por parte de los países en temas ambientales (Corrêa 2006). La lucha ambiental se transforma de local a nacional. Deja de primar la corriente preservacionista, a partir de este momento el objetivo marcado era la creación de un medio rural más autosuficiente, mediante el uso de tecnologías más respetuosas, técnicas apropiadas al medio y socialmente controladas.

Tras la publicación del informe Brundtland (1987), nace el concepto de desarrollo sostenible, que se une a las políticas medioambientales y provoca un cambio tanto de enfoque como de forma de pensar, quedando esto patente en la cumbre de Río de Janeiro en 1992 (cuadro 1). Esta obtuvo un éxito sin precedentes, sobre todo en cuanto a poder de convocatoria, algo inaudito hasta ese momento en temas relacionados con la conservación (Jankilevich 2003). La conservación se sitúa dentro del contexto mundial, y se une a los graves problemas de la humanidad, principalmente al hambre y a la destrucción ambiental del planeta (Tolón y Lastra 2008). Destaca que para obtener una conservación mundial hay que reducir la desigualdad entre los diferentes países (Jankilevich 2003). Se entiende que hasta que la sociedad mundial no tenga acceso a unos ingresos económicos que cubran sus necesidades básicas no se construirá una conciencia ambiental real, algo que era patente en gran parte de la sociedad brasileña hasta hace muy poco tiempo (Corrêa 2006).

\section{EL CASO BRASILEÑO}

En Brasil, las políticas de conservación son creadas a partir de las presiones ejercidas por parte de los Estados Unidos y los países de la Unión Europea sin tener en cuenta que la situación social, económica y ambiental era diferente (Corrêa 2006). Así, se adoptaron leyes ambientales de conservación sin existir una gran preocupación ambiental real por parte de la sociedad o de la clase política.

El desarrollo de los movimientos ambientales en Brasil se divide en tres etapas. El primer periodo comprende

Cuadro 1. Pensamientos ambientales tras Yellowstone y la cumbre de Río de Janeiro.

Yellowstone and environmental thoughts after the Rio de Janeiro conference.

\begin{tabular}{ll}
\hline \multicolumn{1}{c}{ Espíritu de Yellowstone } & \multicolumn{1}{c}{ Espíritu de Río de Janeiro } \\
\hline Santuario natural & Integración en un sistema abierto \\
Protección & Conservación y desarrollo sostenible \\
Estático & Dinámico \\
Gestión centralizada & Gestión descentralizada: ámbito local \\
Regulación & Planificación integral \\
Gestión aislada & Coordinación y cooperación \\
Elitista & Participación social \\
Reacción al desarrollo & Convergencia de objetivos: compatibilidad \\
Excluyente con respecto a otros sectores & Complementario \\
Superficie reducida y delimitada & Superficie amplia, con zonas de transición \\
\hline
\end{tabular}

Fuente: Tolón y Lastra (2008). 
desde 1970 a 1980 y destaca por el dominio de la corriente preservacionista, siendo la creación de parques naturales la principal y única herramienta ambiental. La segunda etapa va de 1980 a 1990. En estos años nace un principio de entendimiento entre los movimientos sociales y ambientales. El tercer y último intervalo va desde 1990 hasta la actualidad (Jacobi 2003). Resalta por la presencia de la corriente socioambiental altamente influenciada por las exigencias de los siringueros ${ }^{2}$, que lleva consigo, entre otras medidas, la creación de las Reservas Extrativistas (Menezes y Siena 2010).

Primer periodo (1970 a 1980). La conferencia de Estocolmo de 1972 fue la principal propulsora de los movimientos ambientales a nivel mundial. A pesar de ello, esta nueva concepción no fue bien aceptada por los países en desarrollo, tras las críticas ambientales lazadas por los países más desarrollados. En Brasil, por ejemplo, interpretan este nuevo enfoque como una crítica a su modelo económico, por lo que dio lugar a la creación de políticas que vendían un respeto ambiental inexistente en la realidad, y empleando la corriente preservacionista como única política ambiental (Menezes y Siena 2010).

Es importante destacar que la cumbre de Estocolmo 1972 no era solo una cumbre sobre medio ambiente para los países en desarrollo: Brasil no era famoso por sus medidas ambientales, era más famoso por el tamaño de la población, así como por la desigualdad regional y la injusticia social (Corrêa 2006). Para Brasil, la cumbre de Estocolmo tenía una gran importancia económica y política, al igual que para otros países en desarrollo, dando como resultado una fuerte discusión entre países desarrollados y países en desarrollo (Jankilevich 2003). Para poder hacerse una idea, en esos años, Brasil estaba bajo un régimen militar con una idea de crecimiento económico que no tenía en cuenta el crecimiento democrático, los derechos humanos o la conservación de la naturaleza (Corrêa 2006).

Segundo periodo (1980 a 1990). Esta segunda etapa destaca por la incorporación de temas ambientales dentro de los discursos de los movimientos sociales. La clase media fue creciendo en número, siendo cada vez mayor el porcentaje de población con los requisitos mínimos cubiertos y estos cambios fomentaron que la sociedad brasileña se preocupara por el medioambiente. De esta manera, los grupos ambientalistas encontraron su sitio en diferentes estratos sociales, altamente apoyados por la clase media brasileira. Paulatinamente se fueron institucionalizando, lo que propició la consecución de una mayor presión política y la inclusión de un capítulo sobre cuestiones ambientales en la

Los siringueros son las personas encargadas de extraer el látex de las siringas y viabilizar su transformación en caucho natural. Los primeros seringueros llegaron a la Amazonía por el año 1870. Trabajaban extrayendo el látex de las seringas por un precio muy reducido, pagado por el dueño de estas, y quedando endeudados por diferentes conceptos haciendo casi imposible el regreso a sus casas (Barbosa 2012). La siringa es un árbol de la familia Euphorbiaceae (Hevea brasiliensis Johannes Müller Argoviensis).
Constitución de 1988, lo que otorgó a estos movimientos un mayor poder legal (Alonso et al. 2007). Además, durante este periodo, destaca la obligación de crear estudios de impacto ambiental y se comienza a sancionar a los agentes agresores del medio ambiente (Jacobi 2003).

Tercer periodo (a partir de 1990). Previamente a este periodo es importante destacar una publicación de finales de los años 80 que es fundamental para ayudar a comprender el cambio de mentalidad que se produjo a partir de los 90 en temas ambientales: Our Common Future, más conocido como informe Brundland (Brundtland 1987), define por primera vez el concepto de desarrollo sostenible, y destaca la importancia que el desarrollo no solo tenga en cuenta los conceptos económicos, sino también sociales y medio ambientales. Tras la cumbre de Río de Janeiro de 1992 se consolidan los movimientos ambientales que buscan un desarrollo sostenible, respaldados por los científicos y empresarios (Jacobi 2003). Así, durante esta etapa el ambientalismo adopta un carácter más económico, y pasa de ser considerado un tema nacional a serlo global. De esta manera, el concepto de desarrollo sostenible sirvió para unir definitivamente a dos grupos separados históricamente, como eran los grupos sociales y los movimientos ambientales (Alonso et al. 2007).

A finales de la década de los 90 comenzó a ganar fuerza la corriente socio-ambiental, altamente influenciada por antiguos líderes siringueros como Chico Mendes o Chico Ginu, que defendían la idea y la posibilidad de conciliar la preservación del medio ambiente y la permanencia de las poblaciones tradicionales (Menezes y Siena 2010). Originalmente, la corriente socio-ambiental no nace como un movimiento ambiental, sino a partir de los movimientos sociales originados por la lucha de las poblaciones tradicionales contra las empresas forestales en relación con la protección de la selva amazónica, vital para la supervivencia de las poblaciones locales. Esto dio como resultado que la lucha por el acceso a los recursos y del territorio se transformara en los principios de la corriente socio-ambiental.

\section{UNIDADES DE CONSERVACIÓN DE BRASIL}

En Brasil las unidades de conservación forman parte del Sistema Nacional de Unidades de Conservación de la naturaleza y pueden ser de tres tipos administrativos: federal, estatal o municipal (Brasil 2000). El organismo público encargado de gestionar las unidades de conservación federal es el Instituto Chico Mendes de Conservación de la Biodiversidad (ICMBio) creado en el año 2007. Este instituto adquirió, además de sus funciones, parte de las funciones acumuladas por el Instituto Brasileiro de Medio Ambiente y de los Recursos Naturales Renovables (Ibama). Su creación se dio en un escenario de conflicto político, sin un planeamiento estratégico profundo de diálogo interno o con la sociedad (Veríssimo et al. 2011).

Las unidades de conservación están definidas como espacios territoriales, que incluyen recursos ambientales 
y aguas jurisdiccionales de características naturales relevantes. Legalmente están instituidas por el poder público y tiene objetivos de conservación y límites bien definidos (Brasil 2000). En función del uso permitido dentro de ellas, se clasifican en: las unidades de conservación de protección integral o de uso indirecto, y las de uso sostenible o de uso directo (Rylands y Brandon 2005). Las primeras tienen como objetivo la preservación de la naturaleza sin contemplar el uso de los recursos naturales. Las segundas, no obstante, tienen el objetivo de compatibilizar la conservación de la naturaleza con el empleo sostenible de los recursos naturales del área delimitada (Brasil 2000). Cada tipo de unidad de conservación puede clasificarse en diferentes categorías (cuadro 2) que engloban diferentes usos y normas.

La creación de áreas de conservación de protección integral en Brasil es habitualmente sinónimo de conflicto con poblaciones tradicionales, al igual que en otros países de América del Sur. El $86 \%$ de los parques naturales existentes en América del Sur tiene poblaciones habitando en su interior (Rios 2004). No obstante, como ya se comentó al inicio del presente trabajo, este estudio se centrará en las unidades de conservación de uso sostenible, destacando las Reservas Extrativistas como las más representativas.

Las unidades de conservación de uso sostenible son las precursoras de las prácticas actuales de la conservación de los recursos naturales. Tienen como base la relación histórica de las poblaciones tradicionales con la naturaleza, por lo tanto, la participación de las comunidades que residen en las áreas es fundamental para poder cumplir los objetivos marcados (Silva y Souza 2009). Fueron creadas por tres razones principales: la primera, nace ante la necesidad de ordenar el territorio y combatir la desforestación ilegal; la segunda, fue por la urgencia de proteger las regiones con alto valor biológico; y la tercera y última razón, se da por la necesidad de atender las demandas de las poblaciones tradicionales y de producción forestal sostenible (Veríssimo et al. 2011).

En las unidades de conservación de uso sostenible es permitido el turismo, la educación ambiental y la extrac- ción de productos forestales madereros y no madereros, siempre que se trate de un manejo sostenible y de acuerdo con el plan de manejo de la unidad. Las poblaciones denominadas tradicionales pueden permanecer en su interior, siempre y cuando realicen actividades que vienen reflejadas en el plano de manejo (Veríssimo et al. 2011). Las comunidades participan en la administración de los parques y reservas creadas (Diegues 2000). Por tanto, las poblaciones tradicionales son vitales para la obtención del objetivo marcado en las áreas protegidas.

Dentro de las unidades de conservación de uso sostenible, como se ha indicado anteriormente, destacan las Reservas Extrativistas, que a su vez pueden ser de dos tipos: terrestres o marinas. Las Reservas Extrativistas son el resultado del conflicto entre dos modelos diferentes de desarrollo, apropiación de tierra, derechos y racionalidad (Cunha y Loureiro 2009). Así, los movimientos sociales, formados por siringueros, lucharon para el mantenimiento de las condiciones de vida existentes en sus territorios, ante las actividades de las empresas madereras que les privaban de elementos básicos para la supervivencia como eran las siringas. En esta lucha no estaba en disputa la tierra, sino la vida misma, ya que estaban en juego los recursos forestales como medio de producción. No era una lucha por el concepto abstracto de la naturaleza, luchaban por su importancia en la vida (Allegretti 2008). El resultado de este conflicto fue la transformación del derecho individual de propiedad al derecho colectivo, por lo que se garantizó el uso de la tierra y el empleo de un método de extracción de recursos más respetuoso con el medio.

Sin embargo, las unidades de conservación de uso sostenible también están asociadas a conflictos políticos, legales, sociales, económicos y ambientales (Silva y Souza 2009). Si bien es cierto que su creación viene precedida de estudios técnicos y consulta pública, para determinar su localización, sus dimensiones y los límites más adecuados (Brasil 2000), rara vez se obtiene un consenso entre todas las partes, y -por lo tanto- se generan conflictos de diferentes grados de intensidad.

Cuadro 2. Categorías de las unidades de conservación.

Categories of conservation units.

\begin{tabular}{ll}
\hline \multicolumn{1}{c}{ Unidades de conservación de protección integral } & \multicolumn{1}{c}{ Unidades de conservación de uso sostenible } \\
\hline Estación Ecológica & Áreas de Protección Ambiental \\
Reserva Biológica & Área de Relevante de Interés Ecológica \\
Parque Nacional & Floresta Nacional \\
Monumento Natural & Reserva Extrativista \\
Refugio de Vidas Silvestres & Reserva de Fauna \\
& Reserva de Desenvolvimiento Sostenible \\
& Reserva Particular de Patrimonio Natural \\
\hline
\end{tabular}

Fuente: Brasil (2000). 


\section{PROBLEMAS Y CONFLICTOS A DESTACAR}

La creación de las unidades de conservación de uso sostenible ha generado menos problemas que sus predecesoras de protección integral, al permitir el uso directo de los recursos por parte de las poblaciones tradicionales. Sin embargo, esto no quiere indicar que están exentas de problemas. Por ejemplo, una deficiente zonificación puede suponer una fragmentación de los hábitats y un tamaño de área insuficiente para poder ubicar a la población. De los diferentes conflictos es importante destacar los problemas originados por la mala aceptación, por parte de antiguos propietarios de las tierras, que ven cómo se les expropian sus tierras y se les limitan los derechos de propiedad (Silva y Souza 2009).

Casi el $30 \%$ de las unidades de conservación creadas en Brasil eran tierras expropiadas a antiguos propietarios latifundistas. Estos reaccionaron, a menudo trataron de impedir la expropiación y limitaron la entrada de las comunidades a los nuevos propietarios.

En ocasiones, otros problemas son generados por una deficiente organización por parte de los miembros de las unidades de conservación o el incumplimiento de las normas reguladoras. Un ejemplo de ello es la apertura de carreteras por parte de los actores locales, de forma irregular, que son aprovechadas por personas no autorizadas y que facilitan las actividades ilegales como la desforestación. $\mathrm{O}$ también crean conflictos entre los miembros de la comunidad por el acceso a tierras que se encuentran próximas a esas carreteras ilegales (Veríssimo et al. 2011).

\section{UNIDADES DE CONSERVACIÓN DE LA UNIÓN EUROPEA}

En 1992 se crea en la Unión Europea la Red Natura 2000, que responde a la necesidad de aprobar una política común sobre las áreas de conservación, pudiendo ser estas compatibles con el mantenimiento de la actividad humana o no. Esta red es considerada como una red ecológica de áreas, cuyo objetivo es garantizar la biodiversidad por medio de la conservación de los hábitats naturales, de la fauna y la floresta silvestre dentro del territorio de los Estados miembros de la Unión Europea, teniendo en cuenta las existencias económicas, sociales y culturales, así como las exigencias particulares de carácter regional y local (CCE 1992).

Se puede definir como una red ecológica europea que nace ante la degradación de los hábitats naturales y el aumento de especies que están en seria amenaza de extinción. Con respeto a esto último, cabe puntualizar que las amenazas de extinción se caracterizan por tener un carácter transfronterizo que hace necesaria la creación de medidas comunitarias con un objetivo final de conservación, implicando a todos los Estados miembros de la Unión Europea (CCE 1992). La Red Natura se enmarca dentro de un nuevo modelo de conservación que tiene como base la creación de redes funcionales de espacios naturales que permitan una relación entre sí y la conservación de la biodiversidad, unidos todos ellos al desarrollo de actividades económicas (Herrero 2008, Olmedo y Gomez 2014).

Dentro de la Red Natura, es de destacar el papel de los corredores ecológicos, cuya función principal es la de conectar las diferentes áreas de conservación y facilitar la movilidad de los seres vivos, evitando el aislamiento y la fragmentación de los hábitats (Bennet 2004). Con la creación de los corredores ecológicos se busca crear una conectividad funcional para recuperar los paisajes fragmentados, por: el crecimiento urbanístico, la creación de infraestructuras o la intensificación agraria. Una buena conectividad ayuda a mantener a las especies silvestres sensibles a la fragmentación del hábitat unido a otros factores como la cantidad y calidad del hábitat disponible (Gurrutxaga 2014).

Para conseguir esto la Red Natura utiliza un procedimiento unitario de selección de lugares, desde una perspectiva Europea y no nacional (Mulero 2004). No obstante, es importante destacar que la legislación que regula la Red Natura no sustituye a ninguna otra legislación de conservación, por lo que puede integrar otras áreas de conservación como son las Reservas de la Biosfera o Parques Nacionales. Así, la Red Natura puede englobar a las demás áreas de conservación y las nuevas creadas.

La Red Natura no es la única política de conservación dentro del contexto europeo, pero sí es la única común a todos los países miembros. Así, las otras políticas de conservación existentes dentro de la Unión Europea son leyes medioambientales propias de cada país miembro. Un ejemplo de la heterogeneidad y disparidad existente dentro de dicha comunidad son las áreas de conservación propias de España, diferentes a otro país miembro de la Unión Europea, y que pueden estar o no, integradas dentro de la Red Natura. No solo eso, en España, las Comunidades Autónomas $^{3}$ tienen la competencia de declarar, planificar y gestionar los espacios protegidos a través de leyes medioambientales propias (Vacas 2005). A mayores otros organismos administrativos locales (municipios) pueden declarar y gestionar espacios protegidos de ámbito local.

Una de las principales características de la Red Natura es la forma de definir y delimitar las áreas a conservar. Estas áreas son propuestas por los propios países miembros de la Unión Europea y no por la Comisión Europea. Así, cada país miembro define las áreas que van a pertenecer a la Red Natura y elaboran un listado de Lugares de Interés Comunitario (LIC) que serán evaluados posteriormente por la Comisión Europea con el fin de comprobar si cumplen con los requisitos Directiva de Hábitats. Tras el proceso de evaluación, y una vez que sean aprobadas y aceptadas para formar parte de esta red, serán consideradas como Zonas Especiales de Conservación. Estas pueden ser

España está dividida en 17 comunidades autónomas y dos ciudades autónomas. Las comunidades autónomas son entidades territoriales administrativas que están dotadas de cierta autonomía legislativa, con representantes propios y determinadas competencias ejecutivas y administrativas. 
de dos tipos: Zonas de Especial Conservación $(\mathrm{ZEC})^{4}$ y Zonas de Especial Protección para las Aves (ZEPA) $)^{5}$. Del mismo modo, las zonas de conservación pueden ser de uso directo o de uso indirecto, según lo defina el propio Estado miembro de la Unión Europea (BOE 2007).

Así, el régimen de protección, las medidas de conservación y la gestión de las diferentes áreas, son reguladas por los países miembros de la Unión Europea, por lo que no es de extrañar que otras figuras de conservación, definidas por cada país, formen parte de la Red Natura (Herrera 2011).

Finalmente, vale la pena destacar que cumplir con las metas de la Red Natura es uno de los mayores retos de la Unión Europea en su objetivo de conservar patrimonio, lo que está asociado a los grandes cambios que se han producido en el modelo de desarrollo rural. El objetivo final es que las áreas definidas puedan constituirse como modelos de desarrollo sostenible, y permitan compatibilizar el desarrollo socioeconómico de las poblaciones rurales con la conservación de la biodiversidad (Vacas 2005). Un ejemplo ilustrativo del uso de la Red Natura para la protección y conservación de especies en peligro es el caso del tejo (Taxus baccata L.) que se puede encontrar en el trabajo de Olmedo y Gomez (2014).

La adaptación de los Estados miembros de la Unión Europea a la Red Natura no fue inmediata, sino un proceso que se prolongó durante un intervalo de años. Un claro ejemplo de ello, es el proceso de adaptación de las leyes medioambientales de España a la Red Natura, que se detalla a continuación.

\section{ADAPTACIÓN DE LA RED NATURA 2000 EN ESPAÑA}

Según los datos publicados por la Comisión Europea (2010), España tiene un 27,20 \% de su superficie dentro de la Red Natura, lo que la sitúa como el cuarto país con mayor porcentaje de superficie. Esto es así debido a la gran diversidad de hábitats y taxones de flora y fauna, $\mathrm{y}$ al alto porcentaje de especies existentes en el territorio Español que se encuentran dentro de los anexos de la Directiva Hábitats (Herrero 2008).

La adaptación de la normativa de Red Natura en España fue un proceso histórico complejo. Como ya se comentó, la Red Natura nació como una medida ambiental donde la definición de las áreas a conservar es realizada de común acuerdo entre el Estado y la Comisión Europea. Pero, dado

\footnotetext{
La finalidad de estos espacios es la de proteger y conservar los hábitats de interés comunitario debido a sus rarezas y su valor ecológico y que están definidos mediante la Directiva Hábitats 92/43/CEE (1992). A mayores están destinados a la protección de especies de flora y fauna de interés por su singularidad e importancia que tienen en la concepción de espacio natural silvestre (Herrera 2011).

Zonas de importancia para la conservación de aves dentro de la Unión Europea, tanto por su importancia en los ciclos de reproducción, por ser zonas de alimentación o zonas estratégicas de migración. Está regulada por la directiva 2009/147/CE (2009) (Herrera 2011).
}

que en España las Comunidades Autonómicas cuentan con competencias en materia ambiental, el Estado actúa como intermediario entre dichas Comunidades Autónomas y la Comisión Europea a la hora de definir y gestionar las diferentes áreas. Esto trajo consigo un considerable grado de heterogeneidad de actuaciones entre unas comunidades y otras, dejando un panorama muy complejo en cuanto a leyes de protección ambiental (Mulero 2004). Así, uno de los principales motivos de esta descoordinación surgió por el proceso de adaptación de la normativa de la Red Natura a la normativa española, lo que no fue una adaptación inmediata, sino desordenada y desorganizada (cuadro 3).

Como se puede comprobar en el cuadro 3, desde la creación de la Red Natura, en el año 1992 hasta su completa regulación en España en el año 2007, pasaron 15 años. Interesa destacar que ante los vacíos legales que hubo en las diferentes etapas, las Comunidades Autónomas crearon sus propias leyes de conservación, incluyendo la normativa de la Red Natura a ritmos muy diferentes y dando como resultado un panorama muy heterogéneo entre las diferentes normativas de conservación (Herrero 2008).

\section{PROBLEMAS Y CONFLICTOS A DESTACAR}

Gran parte de lo que tiene que ver con la conservación y preservación en la Unión Europea, está asociada a las limitaciones en los derechos de la propiedad privada. En este contexto la desconfianza, la falta de interés o de información son algunas de las razones por las que la Red Natura es objeto de rechazo por parte de la población.

La Red Natura debe delimitar y gestionar los espacios, teniendo en cuenta a la población y sus circunstancias sociales y económicas. Pero la realidad es bien distinta, en muchas ocasiones, ya que a la hora de delimitar los espacios se tiene poco en cuenta a la población y se centran principalmente en los valores ambientales de la zona. La dificultad principal viene dada a la hora de declarar los diferentes LIC.

En el proceso de delimitación de las áreas, los países miembros de la Unión Europea también incluyen territorios de propiedad privada dentro de la Red Natura, por lo que se limitan los derechos de los propietarios de esas tierras sin que estos reciban compensación directa por ello, una reducción de impuestos o una bonificación por su ayuda ambiental. De recibir algún tipo de ayuda esta sería indirecta y no vinculada a la Red Natura, sino a aquellos que sean beneficiarios de la Política Agraria Común (Parlamento Europeo 2014).

Por otro lado, aunque la Red Natura no prohíbe ninguna actividad agrícola, sí puede exigir un cambio de la forma de producción. Por ejemplo, un sistema ganadero de uso intensivo puede ser evaluado y exigido a reconvertirse en un sistema más extensivo. Así, algunas de las normas aplicadas y aceptadas por la población, son rechazadas por algunos sectores agrícolas y ganaderos, generando también conflictos. 
Cuadro 3. Cronología del proceso de adaptación de la normativa de la Red Natura 2000 a la normativa española.

Timeline of the process of adapting the rules of Natura 2000 network to the Spanish rules.

\begin{tabular}{lcl}
\hline \multicolumn{1}{c}{ Normativa } & Fecha & \multicolumn{1}{c}{ Observaciones } \\
\hline $\begin{array}{l}\text { Directiva 79/409/CEE relativa a la conservación de las } \\
\text { aves silvestres }\end{array}$ & 1979 & $\begin{array}{l}\text { Normativa Europea. Creación de la Red ZEPA. Primer } \\
\text { paso para la conservación de redes en Europa. }\end{array}$ \\
\hline $\begin{array}{l}\text { Ley estatal 4/89 de Conservación de los espacios naturales } \\
\text { y de la flora y fauna silvestres. }\end{array}$ & 1989 & $\begin{array}{l}\text { Normativa Española. Adaptación de la directiva de Aves } \\
79 / 409 / C E E \text { al ordenamiento interno Español. }\end{array}$ \\
\hline $\begin{array}{l}\text { Directiva 92/43/CEE relativa a la conservación de los } \\
\text { hábitats naturales y de la flora y fauna silvestre, }\end{array}$ & 1992 & Normativa Europea. Nace la Red Natura 2000. \\
\hline $\begin{array}{l}\text { Real Decreto 1997/1995 } \\
\text { Real Decreto 1193/1998 }\end{array}$ & 1995 & $\begin{array}{l}\text { Normativa Española. Adaptación de la Directiva 92/43/ } \\
\text { CEE al ordenamiento interno Español. }\end{array}$ \\
\hline Ley 43/2003 de Montes & $\begin{array}{l}\text { Normativa Española. Modifica el Real Decreto 1997/1995 } \\
\text { al no haber adaptado con exactitud ciertos artículos de la } \\
\text { Directiva 92/43/CEE. }\end{array}$ \\
\hline Ley 43/2007 de Patrimonio Natural y de la Biodiversidad & 2007 & $\begin{array}{l}\text { Normativa Española. Modifica la ley 4/89, introduce un } \\
\text { Capítulo relativo a Red Natura 2000. }\end{array}$ \\
\hline
\end{tabular}

Fuente: Herrero Corral (2008).

Otro escollo a destacar viene asociado a la reintroducción de grandes carnívoros en regiones donde habían estado ausentes durante años. El conflicto fundamental viene marcado por la pérdida puntual de ganado y animales domésticos, como consecuencia de los ataques generados por las especies reintroducidas. Pero no solo los ganaderos están en contra de la reintroducción, también los cazadores que se ven amenazados al encontrarse con un competidor. A raíz de esta situación, en 1991 nació un programa europeo conocido con el nombre de Life-Naturaleza (Comisión Europea 2007) que tenía como objetivo facilitar la conservación. Para ello financió la instalación de vallas eléctricas, cursos para la concienciación de la población local y realiza pagos por las pérdidas ocasionadas por los animales reintroducidos, entre otras funciones. A pesar de la introducción de esas medidas los conflictos no cesaron. En algunos casos se convocaron batidas de caza ante el aumento de ataques al ganado y su baja compensación por las pérdidas, dando como resultado conflictos entre los movimientos ecologistas y los ganaderos (Rabadán y Suárez 2008). Un ejemplo de ello es la reintroducción del oso en los Pirineos, que propició la creación de la organización anti-oso en el 2005 (Bergua 2011). En países como Francia y Rumania, las protestas de los propietarios rurales del sector forestal y de la caza han llevado a replantear el tamaño de las áreas a conservar y a pertenecer a la Red Natura (Cabalar et al. 2016).

Diferentes estudios destacan los problemas surgidos en España tras la implantación de la Red Natura (Mulero Mendigorri 2004, Diego y García 2007, Herrero Corral 2008, Cabalar 2010). Dentro de las diferentes dificultades destacan principalmente la falta de comunicación entre la administración pública y la población, tanto local como general, y el desconocimiento de las diferentes formas de financiamiento.

Finalmente conviene señalar que sectores como el de infraestructuras y la construcción han visto cómo aumentaron las trabas y limitaciones, al aumentar el número de áreas de protección. Con respeto a este tema en España, algunas ONG y asociaciones ecologistas denuncian el mal empleo, por parte de las administraciones locales, de las políticas ambientales. Destacan como algunas áreas están estratégicamente creadas para permitir la construcción en los límites o en el interior de las mismas. Este hecho genera problemas con la población local, al observar falta de transparencia y equidad en la aplicación de la normativa mediante el uso de la conservación y del medio ambiente como excusa (Rabadán y Suárez 2008). Esto fomenta el rechazo a la Red Natura al entender que realmente no se prima la conservación del medio ambiente, aun tratándose de casos puntuales y no de situaciones generalizadas.

\section{COMPARACIÓN DE AMBOS MODELOS}

De lo expuesto hasta el momento se deduce que, ante un mismo objetivo, Brasil y la Unión Europea proponen diferentes soluciones. Ambos emplean el concepto de desarrollo sostenible como identidad de sus políticas de conservación, pero Brasil va más allá al añadir el concepto socio-ambiental a las políticas de conservación, por lo que consigue reducir conflictos en la delimitación del territorio.

Esto queda patente en las diferentes definiciones que tanto Brasil como la Unión Europea hacen del concepto de conservación. Así, Brasil introduce la acción humana como medio de recuperación o preservación y resalta la 
importancia del medio para la supervivencia. Por su parte, la definición de conservación que se emplea en la Unión Europea no enfatiza al ser humano, ya que priman valores científicos y ambientales antes que los sociales. Esto da a entender que para algunos casos se tomará en consideración la presencia del ser humano y en otros, no.

Un ejemplo claro de esto último son las Reservas $E x$ trativistas, que nacieron ante el conflicto por el territorio entre los siringueros y los latifundistas. Para el caso europeo nació la Red Natura, que aunque sea una política de conservación de áreas de uso sostenible, puede contener áreas de protección integral.

Tanto en las unidades de conservación de uso sostenible como en la Red Natura, las áreas están delimitadas en base a criterios técnicos y a una consulta pública, teniendo en cuenta el contexto socio-económico de la cada zona. Como ya se comentó anteriormente, en la Red Natura hay casos donde la consulta pública no se tuvo en cuenta o no se realizó, creando problemas con los propietarios de las tierras, al no ser informados de que sus tierras están dentro de la Red Natura. Para el caso de las Reservas Extrativistas, son las propias comunidades las que solicitan la creación de áreas delimitadas, en otras palabras, fueron y son creadas a partir de una demanda social.

La Red Natura está compuesta por áreas públicas y privadas. Las privadas pueden ser de uso compartido o de uso individual. Los propietarios de las tierras observan como pierden derechos sin recibir una compensación. En el caso de las unidades de conservación, poniendo como ejemplo las Reservas Extrativistas, el uso de las áreas es público de uso compartido, aceptando la población, previamente a la creación de la unidad de conservación, las restricciones que conlleva la creación de la Reserva Extrativista.

Antes de la creación de las unidades de conservación las áreas eran privadas, en otras palabras son tierras que han sido expropiadas a sus antiguos propietarios recibiendo, por tanto, una compensación por ello. Las áreas que forman parte de la Red Natura pueden ser de propiedad privada, pero sus propietarios no reciben ninguna compensación económica por parte del Estado, dando como resultado una pérdida de derechos.

Tomando como ejemplo las Reservas Extrativistas, su creación trae consigo una garantía para las poblaciones tradicionales al asegurarles una superficie en donde pueden trabajar y de la que no serán expulsados, siempre y cuando cumplan las restricciones marcadas por el plano de manejo de cada reserva.

En el caso de las áreas de la Red Natura de uso privado, los dueños no pierden la propiedad de sus tierras, solo pierden algunos derechos pero nunca la propiedad, cosa que no pasa en las Reservas Extrativistas, donde el propietario original de las tierras ve como le expropian la tierra. Por lo tanto, el concepto de propiedad entre las Reservas Extrativistas y Red Natura es diferente.

Ambos sistemas de conservación tienen en común un uso sostenible del territorio, realizando actividades que tienen en cuenta el cuidado y la conservación del medio ambiente. En el caso de Brasil, va más allá, son áreas creadas que también busca la preservación de poblaciones indígenas ${ }^{6}$, poblaciones tradicionales y extractivitas.

Por último, se diría que en la Unión Europea sigue predominando el concepto abstracto de la naturaleza, recuperando y preservando aquellas áreas de interés ambiental, primando más las características ambientales, mientras en Brasil, prima la importancia de la naturaleza en la vida intentando conservar los espacios naturales existentes.

\section{CONCLUSIONES}

Las medidas ambientales empleadas, tanto en Brasil como en la Unión Europea, tienen como objetivo la conservación. Un tema que siempre está asociado a problemas, de mayor o menor gravedad.

Las políticas de conservación de la Unión Europa son el resultado de una degradación del medio, mientras que las políticas de conservación de Brasil, en muchos casos, son creadas para proteger la naturaleza; por lo tanto, no nacen por el mismo motivo.

Uno de los grandes problemas detectados es la necesidad de la participación de los actores. Una falta de información puede generar escepticismo y desconfianza, dando como resultado un rechazo a las medidas.

\section{REFERENCIAS}

Allegretti M. 2008. A construção social de políticas públicas. Chico Mendes e o movimento dos seringueiros. Desenvolvimento e Meio Ambiente 18: 39-59.

Alonso Â, C Veleriano, M Debora. 2007. Identidade e estratégia na formação do movimento ambientalista brasileiro. Novos Estudos - CEBRAP 79: 151-167.

Barbosa MA. 2012. A luta dos seringueiros e a criação das reservas extrativistas: os trabalhadores da borracha numa perspectiva histórica. Revista Eletrônica do Centro de Memória Operária e Popular 1.

Bennet AF. 2004. Enlazando el paisaje: el papel de los corredores y la conectividad en la conservación de la vida silvestre. San José, Costa Rica. UICN-Unión. 278 p.

Bergua JA. 2011. El conflicto ocasionado por la introducción de osos en los pirineos. Diferentes interpretaciones de los contratos natural y nacional. Revista Internacional de Sociología (RIS) 69(2): 439- 460.

BOE (Boletín Oficial del Estado, ES). 2007. LEY 42/2007, de 13 de diciembre, del Patrimonio Natural y de la Biodiversidad. No 299. Consultado el 02 de feb. 2015. Disponible en http:// www.boe.es/diario_boe/txt.php?id=BOE-A-2007-21490

Brasil (Presidência de Republica, BR). 2000. Lei No 9.985 de Julho de 2000. Consultado el 15 de nov. 2014. Disponible en http://www.planalto.gov.br/ccivil_03/LEIS/L9985.htm

Brundtland GH. 1987. Report of the World Commission on environment and development: Our common future. UN. New

Para el caso de poblaciones indígenas existen las reservas indígenas gestionadas por el FUNAI. 
York, USA. Oxford University Press. 400 p.

Brunelle C, KL Goodwin. 2013. Corredor Ecológico: análise para uma proposta no vale do Mutua. Acervo de Iniciação Cientifica 2.

Cabalar M, A Martí, D Royé. 2016. Reflexión sobre los problemas de la Red Natura 2000 en Galicia (NW España). Lurralde: Investigación y Espacio 39: 69-88.

Cabalar M. 2010. La insuficiente protección de la Red Natura 2000 en Galicia, peligros e impactos sobre el medio. In Actas de Biogeografía: una ciencia para la conservación del medio (VI congreso español de biogeografía). p. 181-190.

CCE (Consejo de las Comunidades Europeas). 1992. Directiva 92/43/CEE del consejo, de 21 de mayo de 1992, relativa a la conservación de hábitats naturales y de la fauna y flora silvestre. Consultado 20 de Oct. 2016. Disponible en http:// www.boe.es/doue/1992/206/L00007-00050.pdf

Comisión Europea. 2007. Natura 2000. Boletín de naturaleza de la DG de la Comisión Europea. Natura 2000 21. Consultado 20 de oct. 2016. Disponible en http://ec.europa.eu/environment/nature/info/pubs/docs/nat2000newsl/nat21_es.pdf

Comisión Europea. 2010. Natura 2000 Area Calculation «Snapshot» as of December 2010. Consultado 11 de mar. 2015. Disponible en http://ec.europa.eu/environment/nature/natura $2000 / \mathrm{db}$ gis/pdf/area calc.pdf

Corrêa A. 2006. O Brasil e a três conferências ambientais das Nações Unidas. Río de Janeiro, Brasil. Thesaurus Editora. $276 \mathrm{p}$.

Cunha CC, CFB Loureiro. 2009. Reservas extrativistas: limites e contradições de uma territorialidade seringueira. Theomai 20: 169-185.

Diegues AC. 2000. O Mito Moderno de Natureza Intocada. São Paulo, Brasil. Hucitec. 169 p.

Gurrutxaga M. 2014. Categorización de corredores ecológicos en función de se contribución a la conectividad de la Red Natura 2000. Implicaciones para la ordenación del territorio. GeoFocus 14: 68-84.

Herrera JJ. 2011. La Red Natura 2000, su evolución y análisis de la situación actual en España. Memoria de master en regulación económica y territorial. Facultad de derecho. Universidad de Málaga. 79 p. Consultado 15 de jul. 2015. Disponible en http://www.eumed.net/libros-gratis/2013a/1319/

Herrero G. 2008. Configuración de la Red Natura 2000 en España. Análisis comparativo por comunidades autónomas. Anales de Geografía 28: 85-105

Jacobi P. 2003. Movimento ambientalista no Brasil. Representação social e complexidade de articulação de práticas coletivas. In Bibeiro W ed. Patrimonio Ambiental. São Paulo, Brasil. Edusp. 519-543.

Jankilevich S. 2003. Las cumbres mundiales sobre el medioam- biente. Estocolmo, Rio y Johannesburgo. 30 Años de Historia Ambiental. Buenos Aires, Argentina. Universidad de Belgrano. Documento de trabajo. 106. 32 p.

MacArthur R, E Wilson. 1967. The theory of island biogeography. Princeton, USA. Princeton University Press. 205 p.

Menezes DS, O Siena. 2010. Ambientalismo no Instituto Chico Mendes de Consevação da Biodiversidade (ICMBIO) na Amazônia Legal. Revista Organizações \& Sociedade: 479498.

Mulero A. 2004. Iniciativas internacionales para la protección de espacios naturales. Un análisis crítico de su aplicación en España. Documents d'Análisi Geográfica 44: 167-187.

Olmedo JA, J Gomez. 2014. El tejo en el sur de España: análisis geoecológico y propuesta de conservación de una población mediterranea en peligro crítico de extinción. Bosque 35 (1): 23-36.

Parlamento Europeo. 2014. El segundo pilar de la PAC: La política de desarrollo rural. Consultado 22 oct. 2016. Disponible en http:/www.europarl.europa.eu/atyourservice/es/displayFtu.html?ftuId=FTU_5.2.6.html

Rabadán C, L Suárez. 2008. Mitos sobre la Red Natura 2000: respuestas a las dudas más importantes sobre la red europea de espacios protegidos. Madrid, España. WWF/Adena. 33 p.

Rios AVV. 2004. Populações tradicionais em áreas protegidas. In Ricardo F, V Macedo eds. Terras indígenas e unidades de conservação da natureza: O desafio das sobreposições. São Paulo, Brasil. Instituto Socioambiental. p. 78-84.

Rylands AB, K Brandon. 2005. Unidades de conservação no Brasil. Megadiversidade 1(1): 27-35.

Silva M, Souza RM. 2009. Unidades de conservaçao como estratégia de gestão territorial dos rescursos naturais. Terr@ Plural 3(2): 214-259.

Tolón A, X Lastra. 2008. Los espacios naturales protegidos. Concepto, evolución y situación actual en España. M+A. Revista Electrónica de Medioambiente 5: 1-25.

Vacas T. 2005. Los espacios naturales protegidos, figuras de protección en España. Actas del XIX congreso de geógrafos españoles: espacios públicos, espacios privados, asociación de geógrafos españoles. Consultado 22 oct. 2016. Disponible en http://docplayer.es/13519191-Losespacios-naturales-protegidos-figuras-de-proteccion-enespana-1-creacion-de-los-espacios-naturales-protegidosen-espana.html

Veríssimo A, A Rolla, M Vedoveto, S Futada. 2011. Áreas protegidas na Amazônia brasileira avanços e desafios. Belém/ São Paulo, Brasil. Imazon e ISA. 87 p.

Vivacqua M, P Freire Vieira. 2007. Conflitos socioambientais em unidades de conservação. Política \& Sociedade 4 (7): 139-162. 